\title{
Brane Inflation From Rotation of D4 Brane
}

\author{
Yun-Song Piao ${ }^{a, c}$, Xinmin Zhang ${ }^{a}$ and Yuan-Zhong Zhang ${ }^{b, c}$ \\ ${ }^{a}$ Institute of High Energy Physics, Chinese Academy of Sciences, P.O. Box \\ 918(4), Beijing 100039, China \\ ${ }^{b}$ CCAST (World Lab.), P.O. Box 8730, Beijing 100080 \\ ${ }^{c}$ Institute of Theoretical Physics, Chinese Academy of Sciences, P.O. Box \\ 2735, Beijing 100080, China ${ }^{1}$
}

\begin{abstract}
In this paper, an inflationary model from the rotation of D4-brane is constructed. We show that for a very wide range of parameter, this model satisfies the observation and find that regarded as inflaton, the rotation of branes may be more nature than the distance between branes. Our model offers a new avenue for brane inflation.
\end{abstract}

\footnotetext{
${ }^{1}$ Email address: yspiao@itp.ac.cn
} 
Up to date, inflation [1 is the most likely scenario that explains many problems of the standard cosmology, such as the flatness, the horizon and the monopole problems. Although the recent observations strongly support for the predictions of inflation, it remains a successful idea seeking for a convincing theoretical realization. There exist in the literature numerous phenomenological potentials, which give the proper inflationary properties [2], but some fine-tunings are generically required in order to obtain sufficient e-folding number before the end of inflation and to describe the small amplitude of the observed CMB temperature fluctuations.

It is natural to look for the realizations of inflation within the string theory, since it is arguably our only known consistent description of gravity at the shortest distance and highest energy [3]. Recently, the brane inflationary scenario has been proposed [4], in which the inflaton is identified with an interbrane seperation. In the brane/antibrane model [5], the exchanges of closed string modes between branes give the form of inflaton potential, the inflation ends through the condensation of open string tachyon, which corresponds to hybrid inflation model 6]. However, the inflationary scenario based on brane-antibrane attraction and subsequent collision and annihilation is proved to hardly occur [5, 7], since the interaction between brane and antibrane is very strong, which make the inflaton potential very steep. Although the hypercubic compactification was considered, a fine-tuning to the initial condition is required to obtain enough inflation [5, 8. However, the situation is immensely improved when the branes intersect at a small angle in some compact directions [9]. By choosing sufficiently small angle between the branes the interaction can be made arbitrarily weak, which make the inflaton potential very flat. The small angle corresponds to a small supersymmetry breaking, while the brane/antibrane system is the extreme supersymmetry breaking case, in which two branes are with opposite orientation. Thus the inflation may appear naturally in systems that are not so far from supersymmetry ones. There are also some brane inflationary scenario implemented in other ways [10, 11, 12] based on the string theory.

In this paper, a system which consists of two D4 branes intersecting at a very small angle $\theta$ is considered, in which the distance between two D4 brane is much larger than the string length. We regard $\theta$ as inflaton and study the inflation of D4 brane cosmology. Since the brane system will minimize its energy and make branes become parallel, when the compactification radii are fixed, the changing of $\theta$ is mainly connected with that of the homology class of each brane, in which the homology class of this system is still conservative for a well-defined theory. We calculate the amplitudes of the density perturbation and gravitational waves and give some constraints on the parameters of model.

We assume, before beginning, that the moduli, such as the compactification radii and the dilaton, are stabilized by some unknown mechanism at least during the inflationary period. i.e. the evolution of the closed string modes is much slower than that of the open 
string modes ${ }^{2}$.We take, not losing generalization, that the length of all extra dimensions is equal and denoted by $R$.

As the branes move in a compact space, the R-R tadpole must be cancelled for a welldefined theory, this condition is equivalent to the vanishing of the total homology charge of the system. In addition, the final state after brane inflation is two parallel D4-brane far away from each other and does not annihilate, thus the brane cosmology is still in inflationary phase dominated by the tension of D4-branes and can not exit from it. Generally, an extra plane with opposite orientation and tension far away from this two branes system considered can be put, which is not involved the dynamical evolution of the brane system driving inflation, to avoid these problems. The angle of D4-brane wrapping around a torus is determined by the radii of the torus and the homology class of the brane compactified in it. For our model the radii of torus are fixed by hand, thus the rotation of D4-brane is from the changing of its homology class, which is different from the inflation model of Ref. [12]. The D4 branes wrapping one cycle in torus of radius $R$ with the $\left\{n_{i}, m_{i}\right\}$ homological class have angles $\theta_{i}=\arctan \frac{m_{i}}{n_{i}}$. This branes system have a total homological charge $\left\{\left(n_{1}+n_{2}\right),\left(m_{1}+m_{2}\right)\right\}$. Although the R-R charge of the two D4-branes system being considered should be a constant, but this does not mean that the charge of each D4-brane is unchanged. It is this changing that is considered in our model. For simplification, we take $m_{1}, m_{2} \ll n_{1}, n_{2}$, which corresponds very small angles, and $n_{1}, n_{2}$ are constants, thus the changing of homology class of each brane is mainly from $m_{1}, m_{2}$. Focusing on the relative angle $\theta=\left|\theta_{1}-\theta_{2}\right|$ between the branes, no losing generalization, we take $\theta_{1}=\theta$ and $\theta_{2}=0$, which corresponds to $m_{2}=0$. In the small angle approximation $\theta \ll 1$, the initial energy density of this system is given by

$$
\begin{aligned}
V_{i n i} & =T_{4} R\left(\sqrt{n_{1}^{2}+m_{1}^{2}}+\sqrt{n_{2}^{2}+m_{2}^{2}}\right) \\
& \simeq 2 T_{4} L\left(1+\frac{n_{1} \tan ^{2} \theta}{2\left(n_{1}+n_{2}\right)}+\mathcal{O}\left(\theta^{4}\right)\right)
\end{aligned}
$$

where $T_{4}=\frac{M_{s}^{5}}{(2 \pi)^{4} g_{s}}$ is the $\mathrm{D} 4$ brane tension and $2 L=R\left(n_{1}+n_{2}\right)$ is about the length sum of each brane warped around the correspondent cycle. The two branes system would evolve toward the supersymmetry state of lower energy, i.e. $\theta \rightarrow 0$, thus the final state of this system should be two parallel branes with the same total homological charge.

$$
\begin{aligned}
V_{f i n} & =2 T_{4} R \sqrt{\left(\frac{n_{1}+n_{2}}{2}\right)^{2}+\left(\frac{m_{1}+m_{2}}{2}\right)^{2}} \\
& \simeq 2 T_{4} L\left(1+\frac{n_{1}^{2} \tan ^{2} \theta}{2\left(n_{1}+n_{2}\right)^{2}}+\mathcal{O}\left(\theta^{4}\right)\right)
\end{aligned}
$$

\footnotetext{
${ }^{2}$ Relaxing this condition [14, the inflation from the closed string moduli was considered in Ref. [11, 12]. In fact in our model the inclusion of dilaton will make the system driven to the minimum of the run-away potential. The analysis is much more complicated and goes beyond the scope of this paper.
} 
The change of energy between the initial and final state is

$$
\Delta V=V_{f i n}-V_{i n i} \simeq \frac{T_{4} L}{4} \theta^{2}
$$

The interaction potential between the two D4-brane intersecting at a angles $\theta$ can be computed from the exchange of massless closed string modes, which dual to the one-loop vacuum amplitude for the open string [3. The string perturbation theory computing the interaction between branes requires that the weak coupling condition $g_{s} \ll 1$ must be satisfied. In the limitation of large interbrane separation $y \gg l_{s}$ and small angle $\theta \ll 1$, the potential is 9 , 13 .

$$
V(\theta, y)=-\frac{M_{s}^{2}}{8 \pi^{3}} \frac{\theta^{3}}{y^{2}}
$$

Therefore, the total effective potential for the two D4 branes system can be written as

$$
V_{\text {eff }}=\Delta V+V_{\text {int }} \simeq \frac{T_{4} L}{4} \theta^{2}-\frac{M_{s}^{2}}{32 \pi^{3}} \frac{\theta^{3}}{y^{2}}
$$

In the case of $\theta \ll 1, g_{s} \ll 1$ and $y \gg l_{s}$, we have $\left(y l_{s}^{-1}\right)^{2} \gg \theta g_{s}\left(M_{s} L\right)^{-1}$. Thus we see that the first term in the $V(\theta, y)$ is much larger than the sencond term, which is very important to the calculation of model parameters during inflation in the following.

With the potential (5), the 4D effective action for the angle $\theta$ and the distance $y$ between the branes can be written as ${ }^{3}$

$$
S=\frac{M_{p}^{2}}{2} \int d^{4} x \sqrt{-g} R-\frac{1}{2} T_{4} L \int d^{4} x \sqrt{-g}\left(\partial_{\mu} y \partial^{\mu} y+L^{2} \partial_{\mu} \theta \partial^{\mu} \theta\right)+\int d^{4} x \sqrt{-g} V_{e f f}(\theta, y)
$$

where $M_{p}$ is the $4 \mathrm{D}$ Planck mass, which is given by a dimensional reduction $M_{p}^{2}=$ $M_{s}^{2}\left(R M_{s}\right)^{6} g_{s}^{-2}$. The fields $\theta$ and $y$ can be normalized canonically as

$$
\phi=\theta \sqrt{T_{4} L^{3}} \quad \text { and } \quad \psi=y \sqrt{T_{4} L}
$$

Assuming that inflation occurs initially at a small angle and a large distance between the two D4 branes and the fields corresponding to the angle $\theta$ and distance $y$ is spatially homogeneous but time-dependent, following definition of Ref. [15], the slow-rolling parameters are given

$$
\epsilon_{\theta \theta} \simeq \eta_{\theta \theta} \simeq 32 \pi^{4} \frac{\left(M_{s} R\right)^{6}}{\left(M_{s} L\right)^{3} g_{s}} \frac{1}{\theta^{2}} \quad \epsilon_{y y} \simeq 16 \pi^{4} g_{s} \frac{\left(M_{s} R\right)^{2}}{\left(M_{s} L\right)^{3}}\left(\frac{R}{y}\right)^{6} \theta^{2}
$$

\footnotetext{
${ }^{3} \mathrm{~A}$ detail calculation from supergravity is given in Ref. [9]. The dynamical term relevant to the angle $\theta$ is given by small angle approximations.
} 


$$
\eta_{\theta y} \simeq 16 \pi^{5} \frac{\left(M_{s} R\right)^{3}}{\left(M_{s} L\right)^{3}}\left(\frac{R}{y}\right) \quad \eta_{y y} \simeq-48 \pi^{5} \frac{\left(M_{s} R\right)^{2}}{\left(M_{s} L\right)^{2}}\left(\frac{R}{y}\right)^{4} \theta
$$

In the double fields inflation, the involved calculations are very complex [15]. But fortunately, we find that

$$
\begin{gathered}
\frac{\eta_{\theta \theta}}{\eta_{y y}} \simeq-\frac{2}{\pi g_{s}\left(M_{s} L\right)}\left(\frac{y}{l_{s}}\right)^{4} \gg 1 \\
\frac{\eta_{\theta y}}{\eta_{y y}} \simeq-\frac{y}{3 \theta L} \quad \text { and } \quad \frac{\epsilon_{y y}}{\eta_{y y}} \simeq-\frac{1}{3 \pi} \frac{\theta g_{s}}{M_{s} L}\left(\frac{R}{y}\right)
\end{gathered}
$$

and for a very wide range of parameter, we have $\epsilon_{y y}, \eta_{\theta y},\left|\eta_{y y}\right| \ll \eta_{\theta \theta}$. Therefore, during inflation the rolling of $\theta$ is far fast than that of $y$, and in this case, the double fields inflation in this model can be simplified to a simple field inflation corresponding to $\theta$.

The e-folding number during inflation is

$$
N=\int H d t \simeq-\frac{T_{4} L^{3}}{M_{p}^{2}} \int_{\theta_{60}}^{\theta_{e n d}} \frac{V(\theta)}{V^{\prime}(\theta)} d \theta
$$

where $\theta_{60}$ is the field value corresponding to $N \simeq 60$ required when the COBE scale exits the Hubble radius, and $\theta_{\text {end }}$ is the field value at which inflation ends, which is determined by $\eta_{\theta \theta} \simeq 1$. Thus we obtain $\theta_{60} \sim 10 \theta_{\text {end }}$.

The amplitude of the density perturbation is given by [2]

$$
\mathcal{P}_{s} \sim \frac{H^{2}}{\sqrt{T_{4} L^{3} \dot{\theta}}} \sim \frac{10^{2} g_{s}}{\left(M_{s} L\right)\left(M_{s} R\right)^{3}}
$$

and the correspondent spectrum index is

$$
\begin{aligned}
n-1 & \simeq 2 \eta_{\theta \theta}-6 \epsilon_{\theta \theta} \\
& \sim 10^{2} \pi^{4} \frac{\left(M_{s} R\right)^{6}}{\left(M_{s} L\right)^{3} g_{s}} \frac{1}{\theta_{60}^{2}}
\end{aligned}
$$

The amplitude of the gravitational waves produced during inflation is

$$
\mathcal{P}_{g} \sim \frac{H}{M_{p}} \sim \frac{\left(M_{s} L\right)^{\frac{1}{2}}}{\left(M_{s} R\right)^{6}} g_{s}^{\frac{3}{2}} \theta_{60}
$$

If takeing $g_{s} \sim 0.01, M_{s} R \sim 10$ and $M_{s} L \sim 10^{6}$, we obtain $\theta_{60} \sim 10 \theta_{\text {end }} \sim 0.01, \mathcal{P}_{s} \sim 10^{-5}$, $n \sim 0.99$ and $\mathcal{P}_{g} \sim 10^{-8}$, which are a set of consistent value satisfying the CMB observation. In this case, $M_{s} \sim 10^{-4} M_{p}$. We can see $H \sim 10^{-8} M_{p} \ll M_{s}$, which avoids the correction of higher curvature terms in (6) and makes the description of the effective theory reasonable.

Unlike the inflation model of brane/antibrane or branes inflation with fixed angle, in which the distance between the branes corresponding to inflaton shrinks to $\sqrt{\theta} M_{s}^{-1}$, the 
tachyonic mode of the open string stretching between the branes appears, the inflation ends through the tachyon condensation and a more stable configuration forms [16], under our considering model, the $\theta$ rolls down towards smaller value during inflation, when $\eta_{\theta \theta} \simeq 1$, the inflation ends and $\theta$ oscillates about $\theta=0$, the released energy reheats the brane cosmology. We can estimate the reheating temperature $T_{r e h}$ by regard $T_{r e h}^{4}$ as the initial potential energy

$$
T_{r e h} \sim M_{s}\left(\frac{\left(M_{s} L\right) \theta^{2}}{64 \pi^{4} g_{s}}\right)^{\frac{1}{4}}
$$

Taking above value, we obtain $T_{r e h} \sim 10^{15} \mathrm{Gev}$. Since the distance $y$ almost do not change during inflation, there is not the open string tachyon appeared in this model. When $\theta=0$, the interaction between two brane disappears. The final configuration should be two parallel D4-brane far away from each other, which move towards each other at a very small relative velocity produced during $\theta \neq 0$.

In summary, in the approximation of small angle $\theta$ and large interbrane separation $y$, the double fields inflation of $\theta$ and $y$ from the system, which consists of two D4 branes interacting a angle, was studied. We found that the dynamical evolution of $\theta$ is dominated during inflation i.e. when the dynamics of both $\theta$ and $y$ is considered, the $\theta$ inflation may be more nature. Taking $M_{s} L \sim 10^{6}, M_{s} R \sim 10$ and $g_{s} \sim 0.01$, we obtain the reasonable amplitude and tilt of the density perturbation satisfying the CMB observation and the string scale $M_{s} \sim 10^{15} \mathrm{Gev}$, which corresponds to the GUT scale. In this model, there is not the open string tachyon appeared which make inflation end, as in the model of brane/antibrane or branes with fixed angle. The inflation naturally ends at $\eta_{\theta \theta} \simeq 1$ and the released energy reheats the brane cosmology. A rough estimation of reheating temperature is given $T_{\text {reh }} \sim 10^{15} \mathrm{Gev}$. In fact, how the energy is released and whether it radiate into the brane or bulk remain open, a deeper discuss is worth to be done. Finally, we would like to mention that Halyo have proposed a rotating-brane inflation model [17], but our model is in a different brane setup and parameter regime. Our results offer a new avenue for brane inflation.

\section{Acknowledgments}

We would like to thank Miao Li, Ren-Jie Zhang for interesting conversations and comments on our manuscript. We also thank Qing-Guo Huang for discussions. This project was in part supported by NNSFC under Grant Nos. 10175070, 10047004 and 19925523 as well as also by the Ministry of Science and Technology of China under grant No. NKBRSF G19990754. 


\section{References}

[1] A.H. Guth, Phys. Rev. D23 (1981) 347; A.D. Linde, Phys. Lett. B108 (1982) 389; A.A. Albrecht and P.J. Steinhardt, Phys. Rev. Lett. 48 (1982) 1220.

[2] A.R. Liddle and D.H. Lyth, Cosmological Inflation and Large-Scale Structure, Cambridge University Press, 2000.

[3] J. Polchinski, String Theory, Vols 1 and 2, Cambridge University Press, 1998.

[4] G. Dvali and S.-H.H. Tye, Phys. Lett. B450 (1999) 72, hep-ph/9812483.

[5] C.P. Burgess, M. Majumdar, D. Nolte, F. Quevedo, G.Rajesh and R.J. Zhang, JHEP 07 (2001) 047, hep-th/0105204.

[6] A. Linde, Phys. Rev. D49 (1994) 748, astro-ph/9307002.

[7] G. Dvali, Q. Shafi and S. Solganik, hep-th/0105203.

[8] N. Jones, H. Stoica and S.-H.H. Tye, hep-th/0203163.

[9] J. Garcia-Bellido, R. Rabadan and F. Zamora, JHEP 0201 (2002) 036, hep-th/0112147.

[10] S.H. Alexander, Phys. Rev. D65 (2002) 023507, hep-th/0105032 G. Shiu and S.-H.H. Tye, Phys. Lett. B516 (2001) 421, hep-th/0106274 C. Herdeiro, S. Hirano and R. Kallosh, JHEP 0112 (2001) 027, hep-th/0110271; B.-S. Kyae and Q. shafi, Phys. Lett. B526 (2002) 379, hep-ph/0111101; K. Dasgupta, C. Herdeiro, S. Hirano and R. Kallosh, hep-th/0203019, M. Gómez-Reino and I. Zavala C, hep-th/0207278; D. Choudhury, D. Ghoshal, D.P. Jatkar and S. Panda, hep-th/0305104.

[11] C.P. Burgess, P. Martineau, F. Quevedo, G.Rajesh and R.J. Zhang, hep-th/0111025;

[12] R. Blumenhagen, B. Kors, D. Lust and T. Ott, hep-th/0202124,

[13] H. Arfaei and M.M.S. Jabbari, Phys. Lett. B294 (1997) 288, hep-th/9608167.

[14] J. Garcia-Bellido and R. Rabadan, JHEP 0205 (2002) 042, hep-th/0203247.

[15] D. Wands, N. Bartolo, S. Matarrese and A. Riotto, Phys. Rev. D66 (2002) 043520, astro-ph/0205253.

[16] K. Hashimoto and S. Nagaoka, hep-th/0303204, W.-H. Huang, hep-th/0304171; T. Sato, hep-th/0304237.

[17] E. Halyo, hep-ph/0105341; 